\title{
Kinematic Analysis Validation and Calibration of a Haptic Interface
}

\author{
Mehmet İsmet Can Dede ${ }^{1}$, Barış Taner ${ }^{1}$, Tunç Bilgincan ${ }^{1}$, Marco Ceccarelli $^{2}$ \\ ${ }^{1}$ Department of Mechanical Engineering, İzmir Institute of Technology, 35430 İzmir, Turkey \\ candede@iyte.edu.tr, baristaner@iyte.edu.tr, tbilgincan@gmail.com \\ ${ }^{2}$ Laboratory of Robotics and Mechatronics, DICEM, University of Cassino and South \\ Latium, Via Di Biasio 43, 03043 Cassino (Fr.), Italy \\ ceccarelli@unicas.it
}

\begin{abstract}
Initial calibration tests of a novel hybrid-structured kinesthetic haptic device based on an R-CUBE mechanism is presented in this paper. Experimental validation of the kinematics along with the experimental test set-up description is provided for the manufactured R-CUBE mechanism.
\end{abstract}

Keywords: haptics, parallel mechanism, R-Cube, kinematic analysis, calibration

\section{Introduction}

The word Haptic, based on an ancient Greek word, haptesthai, means related with touch (El Saddik 2007). As an area of robotics, haptics technology provides the sense of touch for robotic applications that involve interaction with human operator and the environment (El Saddik 2007). The sense of touch accompanied with the visual feedback is enough to gather most of the information about a certain environment. It increases the precision of teleoperation and sensation levels of the virtual reality (VR) applications by exerting physical properties of the environment such as forces, motions, textures. Currently, haptic devices find use in many VR and teleoperation applications such as computer aided design (Ott et al. 2010), entertainment (Faust and Yoo 2006), (Web 2012), education (Kretz et al. 2005), training (Basdogan et al. 2001), rehabilitation (Broeren et al. 2004), nanomanipulation (Sieber et al. 2008), virtual prototyping (Zhu and Lee 2004) and virtual sculpting (Leu et al. 2005).

Various types of haptic devices are developed, and they are employed in different types of tasks. Especially for accurate teleoperation (Hokayem and Spong 2006) and precision required VR applications (Ferreira and Mavroidis 2006), high precision haptic systems are required with respect to the current commercially 
available haptic devices. In order to meet this precision criterion, a 6-DoF desktop haptic device, HIPHAD v1.0, was studied and constructed previously (Bilgincan et al. 2010).

This paper focuses on the validation of mechanism analysis of the R-CUBE and the initial calibration tests. Next section of the paper is reserved for explanation of the HIPHAD v1.0 properties and kinematics. Later, the experimental test set-up for initial calibration is described. Finally, the test results are provided including the necessary calculation procedure for adaptation of the device in hardware-in-the-loop simulation environment.

\section{Properties of HIPHAD v1.0}

The device is designated to provide feedback signals to determine the pose of the tool handled by the user in space, therefore it is configured as a 6-DoF mechanism. Only point-type of contact is considered therefore, the motion and the force simulating activity of the tool can be grouped as 3-DoF active translational and 3DoF passive rotation. The properties of the device designed are listed in Table 1.

Table 1. Properties of HIPHAD v1.0 Design.

\begin{tabular}{cc}
\hline \multicolumn{2}{c}{ Properties of the design } \\
\hline Sensation Type & Kinesthetic \\
Mechanical Structure & Hybrid \\
Control Structure & Open-Loop Impedance \\
Application Type & Desktop Device \\
DoF of Motion & 6 \\
Type of Contact Simulation & Point-Type of Contact (Forces in 3D) \\
Continuous Exertable Force & $>0.8 \mathrm{~N}$ in all directions \\
Nominal Positional Resolution & $<0.1 \mathrm{~mm}$ \\
Workspace & $120 \mathrm{~W} \times 120 \mathrm{H} \mathrm{x} \mathrm{120} \mathrm{D}$ \\
Footprint & $<200 \mathrm{~mm}^{2}$
\end{tabular}

In open-loop impedance type haptic devices, due to the random motion of the operator, no control can be employed to avoid singular positions. Therefore, motion through singular positions must be restricted. During the design procedure two singularity conditions are encountered and avoided by necessary precautions in design. Information on mechanical design can be found in (Bilgincan et al. 2010). 


\section{Kinematics Analysis of HIPHAD v1.0}

Kinematics of the device can be easily obtained from the sketch representation of the device in Fig. 1 and Fig. 2.

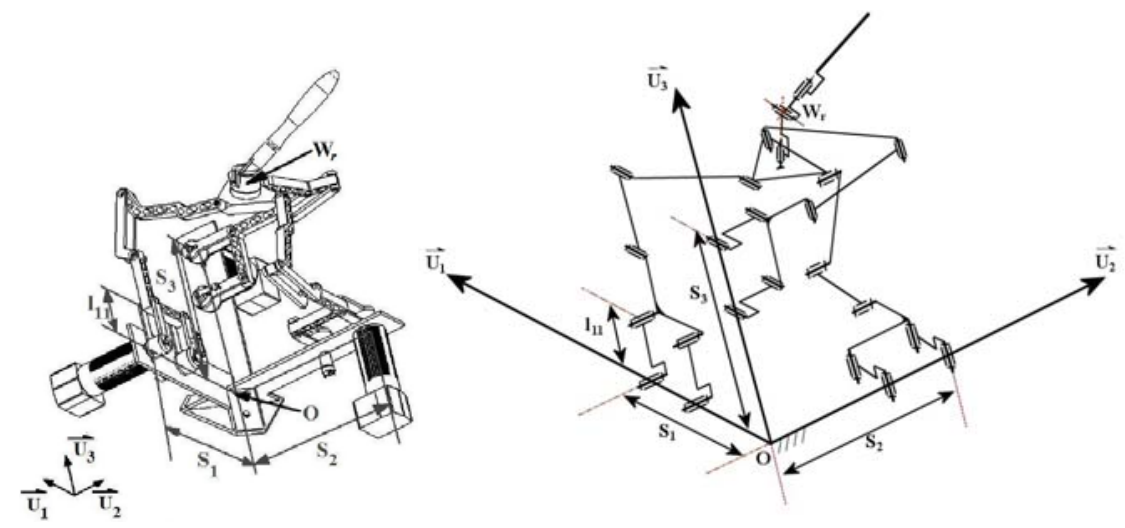

(a)

(b)

Fig. 1 Mechanism parameters: (a) CAD model, (b) sketch view

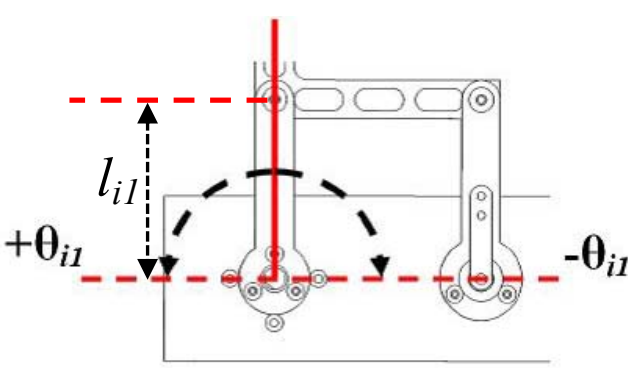

(a)

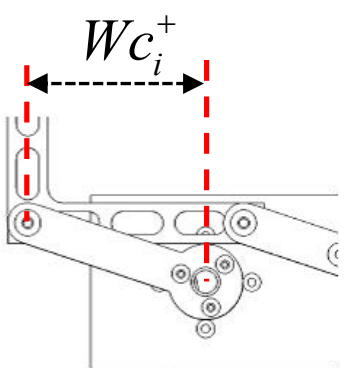

(b)

Fig. 2. (a) Link and joint parameters (b) Joint limit

The translational motion demand send to the slave system is based on the calculation of the $W r$ point by utilizing the real-time measurements from the positions sensors. Thus, direct kinematics is used to calculate the motion demand. In 
Eq. 1, the calculation of the position vector of the tip point, $W r$, with respect to the global origin point, $O$, is described.

$$
\begin{gathered}
\vec{W}_{r}=\sum_{i=1}^{3} W_{r i} \vec{u}_{i} \\
W_{r i}=S_{i}+l_{i} \cdot \sin \left(\theta_{i 1}\right)
\end{gathered} \quad \mathrm{i}=1,2,3
$$

The zero position of the angle, $\theta_{i l}$, is represented with solid red line in Fig. 2(a). $\vec{W} c$ presented in Eq. 2(b) indicates the workspace limitation of the mechanism. Therefore, in Fig. 2, $W c_{i l}$ is theoretically half of the workspace limit along $\vec{u}_{i}$.

$\vec{W} c=\sum_{i=1}^{3} W c_{i} \vec{u}_{i}$

$$
\mathrm{i}=1,2,3
$$

\section{Experimental Test Set-up}

The purpose of this experiment is to first find the absolute measurement of the mechanism's workspace. Later, this information will be transferred into joint space in order to find the joint motion limits. Finally, the joint limits will be matched with joint sensor reading (measured in V) to complete the calibration process.

Workspace is divided into two planes, $\vec{u}_{1}-\vec{u}_{2}$ and $\vec{u}_{1}-\vec{u}_{3}$. On those planes, we are able to measure three displacement values by two cameras, which are parallel to the normal of the selected planes as shown in the Fig. 1 with red arrows. 


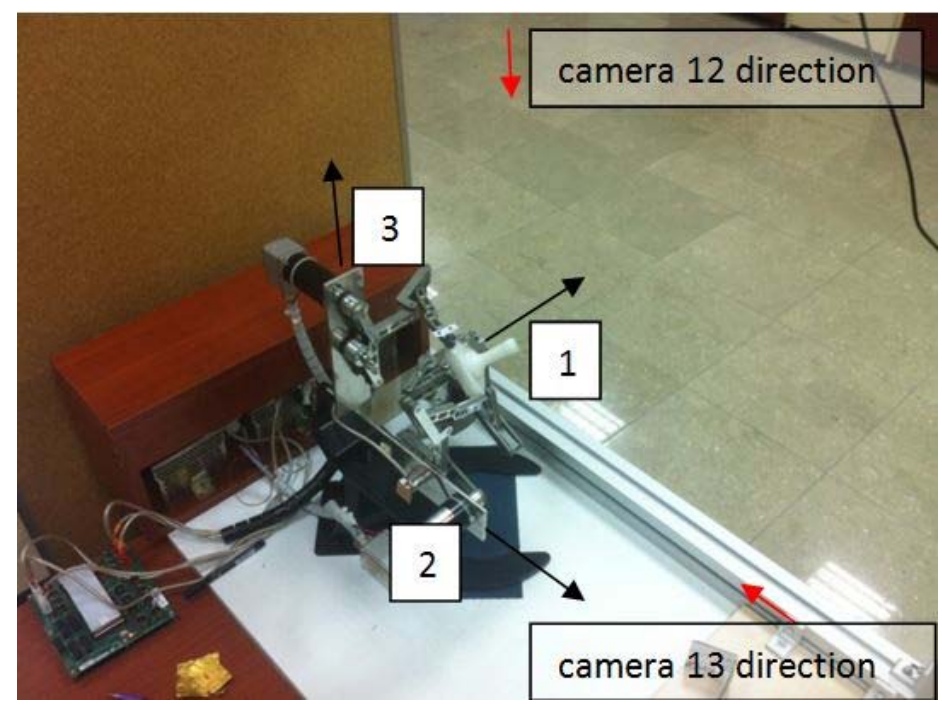

Fig. 3 Experimental test set-up

Cameras are calibrated by finding the distance between two defined circles, whose distance in between their centers are known prior and attached on the end effector's vicinity. The translation of the end effector is measured by 2D-Cross Correlation in Matlab Simulink (Pan et al. 2009).

Total translation of the device along each direction, $W c_{i}$, is divided into two. The two parts are denoted as $W c_{i}^{+}$and $W c_{i}^{-}$representing the positive and negative translations that will be used for calculating $\theta_{i 1}^{+}$and $\theta_{i 1}^{-}$angles.

Test procedure is initiated by finding the mid-position of the joint range, where $\theta_{i l}=0^{\circ}$, with a set-square. Then, the positive and negative limits, which are $W c_{i}^{+}$ and $W c_{i}^{-}$, of the mechanism are measured via cameras. Measured workspace limits are also used for calculating the joint limits $\theta_{i 1}^{+}$and $\theta_{i 1}^{-}$as described in the Eq. 3.

$\theta_{i 1}^{+}=\arcsin \left(W c_{i}^{+} / l_{1 i}\right)$

$\theta_{i 1}^{-}=\arcsin \left(W c_{i}^{-} / l_{1 i}\right)$ 


\section{Test Results}

Mechanism is located at its limits to measure workspace limits. Measured workspace limits and calculated joint limits are tabulated in the Table 2. Workspace limits are given as the mean value for six successive measurements with the same test procedure. Total angle swept by each joint within the workspace is calculated to have mean values of $140.58^{\circ}, 136.50^{\circ}$ and $137.79^{\circ}$ for $\theta_{11}, \theta_{21}$ and $\theta_{31}$ respectively. Standard deviation for value for each joint limit is calculated to be $s_{11}=1.60, s_{21}=1.26$ and $s_{31}=1.47$. According to the statistics of data acquired from six sets of measured displacement values, error bounds are calculated for the $95.45 \%$ of measurement as; first joint limit bounded within $\pm 3.20^{\circ}$ error, second joint limit bounded within $\pm 2.94^{\circ}$ error and third joint limit bounded within $\pm 2.51^{\circ}$ error.

Table 2. Measured Values from Cameras and Calculated Joint Limits

\begin{tabular}{cccc}
\hline Workspace Limits & $\begin{array}{c}\text { Means of Measured } \\
\text { Values (mm) }\end{array}$ & Joint Limits & $\begin{array}{c}\text { Calculated Values } \\
\left({ }^{\circ}\right)\end{array}$ \\
\hline$W c_{1}^{+} / W c_{1}^{-}$ & $60,65 / 61.67$ & $\theta_{11}^{+} / \theta_{11}^{-}$ & $71.64 / 68,94$ \\
$W c_{2}^{+} / W c_{2}^{-}$ & $60.15 / 60,55$ & $\theta_{21}^{+} / \theta_{21}^{-}$ & $68.72 / 67.79$ \\
$W c_{3}^{+} / W c_{3}^{-}$ & $61.22 / 59.99$ & $\theta_{31}^{+} / \theta_{31}^{-}$ & $67.38 / 70.41$ \\
\hline
\end{tabular}

Voltage values measured from the potentiometers in each axis are given in the Table 3 with respect to the axis numbers. These values are measured at the joint limits, indicated with min and max, and also for the mid-position of the workspace, indicated by mid. In addition, the measurement ranges in between the joint limits are provided to be used in constant gain, $K_{i}$, calculation for converting the raw data into measured joint positions by using Eq. 4.

$$
K_{i}=\left(\theta_{i 1}^{+}+\theta_{i 1}^{-}\right) / V r_{i} \quad \mathrm{i}=1,2,3
$$

Finally joint angles at an instant during operation are calculated by the Eq. 5 by using measured voltage from the sensors of the joints, $V m_{i}$ for $\mathrm{i}=1,2,3$.

$$
\left(V m_{i}-\operatorname{Vmid}_{i}\right) \times K_{i}=\theta_{i 1} \quad \mathrm{i}=1,2,3
$$


Table 3. Voltage values Measured from Joint Sensors (Potentiometers)

\begin{tabular}{|c|c|c|c|c|c|c|c|c|}
\hline \multicolumn{3}{|c|}{ Pot $_{1}$} & \multicolumn{3}{|c|}{$\mathrm{Pot}_{2}$} & \multicolumn{3}{|c|}{$\mathrm{Pot}_{3}$} \\
\hline \multicolumn{2}{|c|}{$\begin{array}{l}\text { Voltage Values } \\
\text { (V) }\end{array}$} & \multirow[t]{2}{*}{$\begin{array}{l}\text { Voltage } \\
\text { Range, } \mathrm{Vr} \\
\text { (V) }\end{array}$} & \multicolumn{2}{|c|}{$\begin{array}{c}\text { Voltage } \\
\text { Values (V) }\end{array}$} & \multirow[t]{2}{*}{$\begin{array}{c}\text { Voltage } \\
\text { Range, Vr (V) }\end{array}$} & \multicolumn{2}{|c|}{$\begin{array}{l}\text { Voltage Values } \\
\text { (V) }\end{array}$} & \multirow[t]{2}{*}{$\begin{array}{l}\text { Voltage } \\
\text { Range, Vr } \\
\text { (V) }\end{array}$} \\
\hline $\mathrm{Vmin}_{1}$ & 2,68 & & $\mathrm{Vmin}_{2}$ & 2,20 & & $\mathrm{Vmin}_{3}$ & 0,86 & \\
\hline $\mathrm{Vmid}_{1}$ & 5,33 & $\mathrm{Vr}_{1}=5,07$ & $\mathrm{Vmid}_{2}$ & 5,11 & $\mathrm{Vr}_{2}=5,57$ & $\mathrm{Vmid}_{3}$ & 3,64 & $\mathrm{Vr}_{3}=5,30$ \\
\hline $\operatorname{Vmax}_{1}$ & 7,70 & & $\operatorname{Vmax}_{2}$ & 7,78 & & $\operatorname{Vmax}_{3}$ & 6,17 & \\
\hline
\end{tabular}

\section{Conclusions}

The aim of this work was to calibrate and experimentally validate the kinematics of a novel haptic device, HIPHAD v1.0. External measurement of the absolute position of the mechanism is carried out by using a vision-aided algorithm. Calibration of the mechanism is done in accordance with the test results. Test results also indicate the precision errors in manufacturing and assembling the device which are accounted for by performing the calibration. As a result of the tests, repeatability of the device is calculated to be around $2^{\circ} / 100^{\circ}$. This performance can be improved for applications calling for increased precision by changing the joint sensors with encoder with higher resolutions. Future work includes the calibration of forces exerted by the mechanism to the human operator.

\section{References}

Basdogan C, Ho C-H, Srinivasan MA (2001) Virtual Environments for Medical Training: Graphical and Haptic Simulation of Laparoscopic Common Bile Duct Exploration. IEEE/ASME Trans. Mechatron. 6 (3): 269-285

Bilgincan T, Gezgin E, Dede MIC (2010) Integration of the hybrid-structure haptic interface: HIPHAD v1.0. Proceedings of the International Symposium of Mechanism and Machine Theory

Broeren J, Rydmark M, Sunnerhagen KS (2004) Virtual Reality and Haptics as a Training Device for Movement Rehabilitation after Stroke: A Single-Case 
Study. Arch. Phys. Med. Rehabil. 85 (8): 1247-1250

El Saddik A (2007) The Potential of Haptics Technologies. IEEE Instrum. Meas. Mag. 10 (1): 10-17

Faust M, Yoo Y-H (2006) Haptic feedback in pervasive games. 3rd Int. Workshop Pervasive Gaming Appl.

Ferreira A, Mavroidis C (2006) Virtual Reality and Haptics for Nanorobotics. IEEE Robot. Autom. Mag. 13 (3): 78-92

Hokayem PF, Spong MW (2006) Bilateral Teleoperation: An Historical Survey. Autom. 42 (12): 2035-2057

Kretz A, Huber R, Fjeld M (2005) Force Feedback Slider (FFS): Interactive Device for Learning System Dynamics. IEEE Int. Conf. Adv. Learn. Technol. 457-458

Leu MC, Peng X, Zhang W (2005) Surface Reconstruction for Interactive Modeling of Freeform Solids by Virtual Sculpting. CIRP Ann. - Manuf. Technol. 54 (1): 131-134

Ott R, Vexo F, Thalmann D (2010) Two-handed Haptic Manipulation for CAD and VR Applications. Comput.-Aided Des. Appl. 7(1): 125-138

Pan B, Qian K, Xie H, Asundi A (2009) Two-Dimensional Digital Image Correlation For In-Plane Displacement and Strain Measurement. Meas. Sci. Technol. doi:10.1088/0957-0233/20/6/062001

Sieber A, Valdastri P, Houston K, Eder C, Tonet O, Menciassi A, Dario P (2008) A Novel Haptic Platform for Real Time Bilateral Biomanipulation with a MEMS Sensor for Triaxial Force Feedback. Sens. Actuator. A: Phys. 142 (1): $19-27$

Web, http://www.novint.com/index.php/novintfalcon. accessed on July 27th, 2012

Zhu W, Lee Y-S (2004) Five-Axis Pencil-Cut Planning and Virtual Prototyping with 5-DOF Haptic Interface. Comput.-Aided Des. 36 (13): 1295-1307 